\title{
Pemetrexed induces apoptosis in malignant mesothelioma and lung cancer cells through activation of reactive oxygen species and inhibition of sirtuin 1
}

\author{
KI-EUN HWANG $^{1 *}$, YOUNG-SUK KIM $^{1 *}$, YU-RI HWANG ${ }^{1}$, SU-JIN KWON ${ }^{1}$, DO-SIM PARK ${ }^{2}$, \\ BYONG-KI CHA ${ }^{3}$, BYOUNG-RYUN KIM ${ }^{4}$, KWON-HA YOON ${ }^{5}$, EUN-TAIK JEONG ${ }^{1}$ and HAK-RYUL KIM ${ }^{1}$ \\ ${ }^{1}$ Department of Internal Medicine, Institute of Wonkwang Medical Science and ${ }^{2}$ Department of Laboratory Medicine, \\ Wonkwang University School of Medicine; ${ }^{3}$ Thoracic and Cardiovascular Surgery, Chonbuk National University \\ Medical School; Departments of ${ }^{4}$ Obstetrics and Gynecology, and ${ }^{5}$ Radiology, Wonkwang University \\ School of Medicine, Jeonbuk 570-749, Republic of Korea
}

Received November 5, 2014; Accepted February 9, 2015

DOI: $10.3892 /$ or.2015.3830

\begin{abstract}
Pemetrexed is a multitargeted antifolate used for the treatment of malignant mesothelioma and non-small cell lung cancer (NSCLC). However, the mechanism by which pemetrexed induces apoptosis remains unclear. In the present study, we investigated the involvement of reactive oxygen species (ROS) and sirtuin 1 (SIRT1) in pemetrexed-induced apoptosis in MSTO-211 malignant mesothelioma cells and A549 NSCLC cells. Pemetrexed enhanced caspase-dependent apoptosis, induced intracellular ROS generation, and downregulated SIRT1 in the MSTO-211 and A549 cells. Pemetrexed-induced apoptosis, which was prevented by pretreatment with $\mathrm{N}$-acetylcysteine (NAC), was mediated by effects on the mitochondria, including mitochondrial membrane potential transition (MPT) and cytosolic release of cytochrome $c$, and also involved regulation of SIRT1 expression. Interference with SIRT1 expression using siRNA enhanced pemetrexed-induced apoptosis through mitochondrial dysfunction and ROS generation, whereas resveratrol, an activator of SIRT1, protected against pemetrexed-induced apoptosis. These results show that pemetrexed induces apoptosis in MSTO-211 mesothelioma cells and A549 NSCLC cells through mitochondrial dysfunction mediated by ROS accumulation and SIRT1 downregulation.
\end{abstract}

Correspondence to: Professor Hak-Ryul Kim, Department of Internal Medicine, Institute of Wonkwang Medical Science, Wonkwang University School of Medicine, 344-2 Shinyong-dong Iksan, Jeonbuk 570-749, Republic of Korea

E-mail: kshryj@wku.ac.kr

*Contributed equally

Key words: pemetrexed, apoptosis, reactive oxygen species, sirtuin 1, malignant mesothelioma, lung cancer

\section{Introduction}

Lung cancer is the leading cause of cancer-related death worldwide and is associated with a 5-year survival rate of $15 \%$ (1). Non-small cell lung cancer (NSCLC) accounts for $\sim 80 \%$ of all lung cancers and is associated with relatively low survival rates. Among patients with advanced NSCLC and good performance status, even standard first-line platinumbased chemotherapy is associated with response rates of less than $40 \%$ and a median length of survival of 8-14 months after diagnosis (2). Malignant mesothelioma is an aggressive tumor type with a very poor prognosis and a median length of patient survival of 9-12 months after diagnosis. At this time, there are few effective chemotherapeutic options for the treatment of malignant mesothelioma, yet available treatments include cisplatin, vinorelbin and gemcitabine (3). We believe that new strategies based on better understanding of tumor biology could maximize the efficacy of current treatments.

Pemetrexed, a multitargeted antifolate cytotoxic agent, is used to treat malignant mesothelioma and NSCLC, mainly in nonsquamous cell carcinomas (4-6). Pemetrexed targets enzymes involved in the de novo biosynthesis of purines and pyrimidines, including thymidylate synthase (TS), dihydrofolate reductase (DHFR) and glycinamide ribonucleotide formyltransferase (GARFT), thereby inducing an imbalance in the nucleotide pool and DNA damage (7). Cellular studies indicate that pemetrexed induces DNA damage, growth arrest and apoptosis by generating reactive oxygen species (ROS), accumulation of which contributes to the apoptosis of human melanoma cells treated with pemetrexed (8). On the basis of these results, we hypothesized that pemetrexed has the potential to induce apoptosis in malignant mesothelioma and lung cancer cells via ROS accumulation.

Sirtuin 1 (SIRT1) is a nicotinamide adenine dinucleotide $\left(\mathrm{NAD}^{+}\right)$-dependent deacetylase that exerts its biological effects by deacetylating histones and non-histone proteins, and its substrates include many proto-oncogenes and tumor suppressors (9). Previous studies have reported that SIRT1 is overexpressed and activated in certain types of human 
cancers, and that SIRT1 overexpression blocks apoptosis and senescence, while promoting cell proliferation and angiogenesis (10-12). Inhibition of SIRT1 was found to induce growth arrest and apoptosis in several types of cancer cells $(13,14)$.

The aim of the present study was to examine the effects of pemetrexed in malignant mesothelioma and lung cancer cells and study the mechanisms by which it produces these effects. This is the first study to report the involvement of ROS and SIRT1 in pemetrexed-induced apoptosis in malignant mesothelioma and NSCLC cell lines.

\section{Materials and methods}

Materials. RPMI-1640, fetal bovine serum (FBS), and antibiotics were obtained from Gibco-BRL Co. (Grand Island, NY, USA). Pemetrexed was purchased from Toronto Research Chemicals, Inc. (Toronto, Ontario, Canada). 3-(4,5-Dimethyl2-thiazolyl)-2,5-diphenyl-2H-tetrazolium bromide (MTT), propidium iodide (PI), dimethyl sulfoxide (DMSO), $\mathrm{N}$-acetylcysteine (NAC) and resveratrol were purchased from Sigma-Aldrich (St. Louis, MO, USA). JC-1, a lipophilic fluorescent dye used to detect mitochondrial membrane depolarization, was obtained from Molecular Probes Co. (Eugene, OR, USA). Primary antibodies against the following targets, caspase-3,-8 and -9, and poly(ADP-ribose) polymerase (PARP) were obtained from Santa Cruz Biotechnology (Santa Cruz, CA, USA). Antibodies against SIRT1, voltage-dependent anion channel (VDAC) and glyceraldehyde 3-phosphate dehydrogenase (GAPDH) were purchased from Cell Signaling Technology (Beverly, MA, USA). Antibodies against cytochrome $c$ were obtained from Pharmingen (San Diego, CA, USA). Anti-rabbit IgG-conjugated horseradish peroxidase (HRP) antibodies and enhanced chemiluminescence (ECL) kits were purchased from Amersham Pharmacia Biotech (Buckinghamshire, UK).

Cell culture and viability test. The human mesothelioma cell line MSTO-211H was obtained from the American Type Culture Collection (ATCC; Manassas, VA, USA), and the human lung adenocarcinoma cancer cell line A549 was obtained from the Korean Cell Line Bank (KCLB, Seoul, Korea). These cell lines were grown in RPMI-1640 containing $100 \mathrm{U} / \mathrm{ml}$ penicillin, $0.1 \mathrm{mg} / \mathrm{ml}$ streptomycin and $10 \% \mathrm{FBS}$. The cells were incubated in a humidified atmosphere of $5 \% \mathrm{CO}_{2}$ in air at $37^{\circ} \mathrm{C}$ and maintained in log phase growth. Cell viability was determined by measuring the mitochondrial conversion of MTT to formazan, which was measured spectrophotometrically. After cells were treated with the specified study drugs, MTT was added to the cell suspension for $4 \mathrm{~h}$. After 3 washes with phosphate-buffered saline (PBS; pH 7.4), the insoluble formazan product was dissolved in DMSO. The optical density (OD) of each well was measured using a microplate reader (Titertek Multiskan; Flow Laboratories, North Ryde, New South Wales, Australia) at $590 \mathrm{~nm}$. The OD resulting from formazan production in the control cells was considered as representing $100 \%$ cell viability, and all other measurements were expressed as a percentage of the control cell value.

Annexin $V$ assay for the assessment of apoptosis. MSTO-211 and A549 cells undergoing early/late apoptosis were analyzed by Annexin V-fluorescein isothiocyanate (FITC) and PI staining. Cells in the log phase $\left(2.5 \times 10^{5}\right.$ cells $)$ were seeded in $35-\mathrm{mm}^{2}$ dishes. Cells were left untreated or were incubated with the specified drugs for the indicated times at $37^{\circ} \mathrm{C}$. Both adherent and floating cells were collected and analyzed by the Annexin V assay according to the manufacturer's instructions. Pelleted cells were briefly washed with PBS and resuspended in Annexin binding buffer. Cells were then incubated with Annexin V-FITC and PI for $15 \mathrm{~min}$ at room temperature. After incubation, the stained cells were analyzed using a fluorescence-activated cell sorting (FACS)Calibur system equipped with CellQuest software (Becton-Dickinson, San Jose, CA, USA). Cells with no drug treatment were used as controls.

Measurement of the mitochondrial membrane potential $(\Delta \Psi m)$. MSTO-211 and A549 cells were harvested at the indicated treatment times, washed with PBS, and then stained with $10 \mu \mathrm{g} / \mathrm{ml} \mathrm{JC}-1$ at $37^{\circ} \mathrm{C}$ for $30 \mathrm{~min}$. After a brief wash with PBS, cells were immediately analyzed using a FACSCalibur system equipped with CellQuest software. At low concentrations, JC-1 exists mainly in a monomeric form, emitting green fluorescence with an emission maximum at $\sim 530 \mathrm{nM}$, whereas at higher concentrations it forms aggregates known as J-aggregates, which emit orange-red fluorescence with an emission maximum at $\sim 590 \mathrm{nM}$.

Measurement of ROS. To measure intracellular ROS, cells were incubated with $10 \mu \mathrm{mol} / 1$ 5-(and-6)-carboxy-2',7'dichlorodihydrofluorescein diacetate (carboxy- ${ }_{2}$ DCFDA; Molecular Probes Co.) at $37^{\circ} \mathrm{C}$ for $30 \mathrm{~min}$. Cells were washed, scraped gently, resuspended in PBS and kept on ice for immediate analysis by FACSCalibur flow cytometry using an argon laser (488 nm) for excitation (Becton-Dickinson). Green fluorescence due to DCF trapped inside the cells was measured and plotted on a log scale. Data were acquired and analyzed with the CellQuest program (Becton-Dickinson).

Western blotting. Cells were harvested and lysed using radioimmunoprecipitation assay buffer (50 mM Tris-Cl, $\mathrm{pH} 7.4,1 \%$ NP40, $150 \mathrm{mM} \mathrm{NaCl}, 1 \mathrm{mM}$ EDTA, $1 \mathrm{mM}$ phenylmethylsulfonyl fluoride, $1 \mu \mathrm{g} / \mathrm{ml}$ each of aprotinin and leupeptin, and $1 \mathrm{mM} \mathrm{Na}_{3} \mathrm{VO}_{4}$ ). After centrifugation at 12,000 x $\mathrm{g}$ for $30 \mathrm{~min}$, the supernatant was collected and the protein concentration was determined by the Bradford method (Bio-Rad Protein Assay; Bio-Rad, Hercules, CA, USA). Equal amounts of protein were separated by $12 \%$ sodium dodecyl sulfate-polyacrylamide gel electrophoresis (SDS-PAGE) under reducing conditions and transferred to nitrocellulose membranes. The membranes were blocked with 5\% skim milk in TBS-T (25 mM Tris, $\mathrm{pH} 7.6,138 \mathrm{mM} \mathrm{NaCl}$ and $0.05 \%$ Tween-20) for $1 \mathrm{~h}$ and probed with primary antibodies (at 1:1,000-1:5,000). After a series of washes, the membranes were further incubated with a secondary antibody (at 1:2,000-1:10,000) conjugated with HRP. Detection of the immunoreactive signals was carried out using an ECL detection system.

Preparation of cytosolic and mitochondrial fractions. Cytosolic and mitochondrial fractions were prepared as previously described (15) with modifications. Cells were harvested, washed with ice-cold PBS, and then incubated with $500 \mu \mathrm{M}$ 
buffer A [250 mM sucrose, $20 \mathrm{mM}$ HEPES (pH 7.5), $10 \mathrm{mM}$ $\mathrm{KCl}, 1.5 \mathrm{mM} \mathrm{MgCl} \mathrm{mM}_{2}, 1 \mathrm{mM}$ EGTA, $1 \mathrm{mM}$ EDTA, $1 \mathrm{mM}$ DTT, $1 \mathrm{mM}$ PMSF and $10 \mu \mathrm{g} / \mathrm{ml}$ each of leupeptin, aprotinin and pepstatin A] on ice for $30 \mathrm{~min}$. Cells were then disrupted by 20 passages through a 26 -gauge needle and centrifuged at $750 \times \mathrm{g}$ for $10 \mathrm{~min}$. The supernatant was centrifuged at $10,000 \times \mathrm{g}$ for $25 \mathrm{~min}$. After centrifugation, the cytosolic fraction was frozen at $70^{\circ} \mathrm{C}$. The pellet containing the mitochondria was washed with ice-cold buffer $\mathrm{A}$ and then resuspended with cell lysis buffer. The resuspended pellet was incubated on ice for $30 \mathrm{~min}$ and then centrifuged at $10,000 \mathrm{x}$ g for $25 \mathrm{~min}$. The supernatant thus collected represented the mitochondrial fraction of the cells.

Gene silencing. Transcriptional expression of SIRT1 was specifically suppressed by the introduction of a 21-nucleotide duplex small interfering RNA (siRNA) targeting the coding sequence of SIRT1 mRNA (16). Cells $\left(10^{5}\right.$ cells/well) were plated in 6-well plates and transiently transfected with $50 \mathrm{nM} /$ well of SIRT1 siRNA (Cell Signaling Technology) mixed with the X-tremeGENE siRNA transfection reagent (Roche Applied Science, Penzberg, Germany) according to the manufacturer's instructions. Silencer Negative Control siRNA (Roche Applied Science) was used as a negative control and introduced into the cells using the same protocol.

Statistical analysis. Each experiment was performed at least 3 times, and all values are expressed as the mean \pm SD of triplicate samples. The Student's t-test was used to determine the statistical significance of the results. Values of $\mathrm{p}<0.05$ were considered to indicate statistically significant results.

\section{Results}

Effect of pemetrexed on the apoptotic activity of malignant mesothelioma and lung cancer cells. MSTO-211 and A549 cells were treated with different concentrations of pemetrexed, and the viability was measured by the MTT assay. As shown in Fig. 1A, pemetrexed significantly inhibited the growth of MSTO-211 and A549 cells in a dose-dependent manner. To determine whether the observed growth inhibition was due to enhanced apoptosis, the proportion of apoptotic cells was measured using Annexin V-PI staining. Annexin V staining showed that pemetrexed significantly enhanced apoptosis in the MSTO-211 and A549 cells (Fig. 1B). To further elucidate the mechanism through which pemetrexed induced apoptosis, cell lysates were evaluated by immunoblotting (Fig. 1C). Pemetrexed enhanced the expression of the processed $85-\mathrm{kDa}$ isoform of PARP, which is known to play a major role in the process of apoptosis. Moreover, pemetrexed led to a marked increase in the expression of caspase-3, -8 and -9 . These results indicate that pemetrexed enhanced caspase-dependent apoptosis in the MSTO-211 and A549 cells.

Pemetrexed induces intracellular ROS production and downregulates SIRT1 in malignant mesothelioma and lung cancer cells. We examined the role of signal transduction pathways in modulating pemetrexed-induced apoptosis. Intracellular ROS generation was assessed by flow cytometry using the total ROS marker $\mathrm{H}_{2}$ DCFDA. Elevated ROS levels in the MSTO-211 and
A

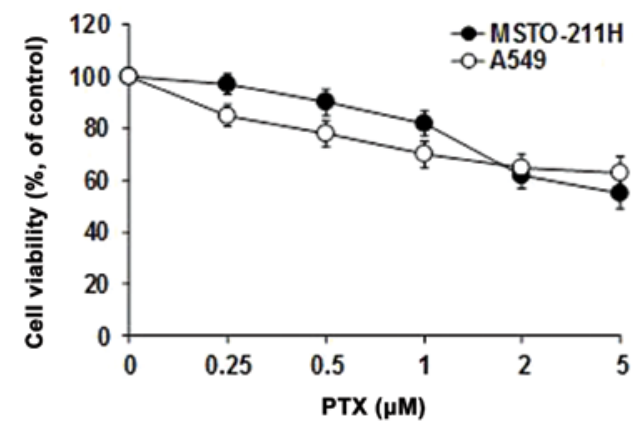

B
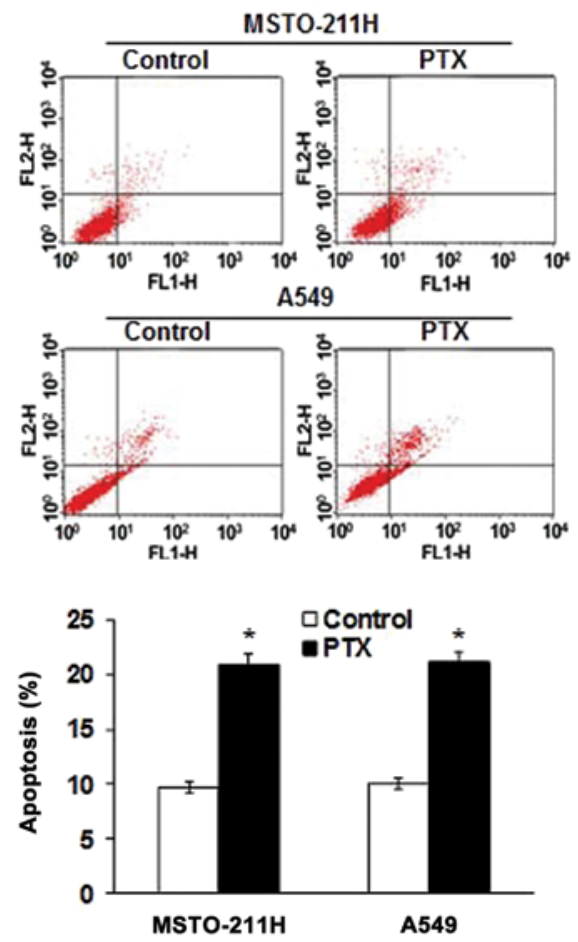

C

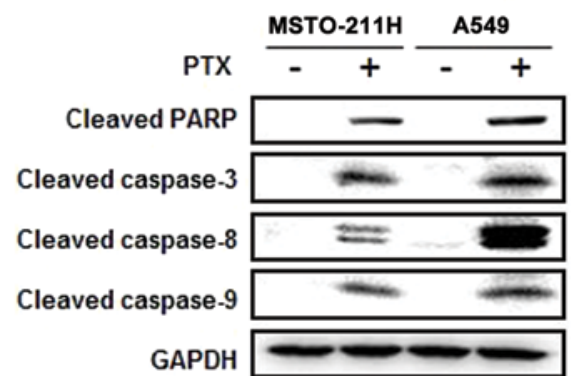

Figure 1. Pemetrexed-induced apoptosis in MSTO-211 and A549 cells. (A) The cells were treated with different concentrations of pemetrexed for $24 \mathrm{~h}$ and viability was measured by the MTT assay. The viability of the control cells was set as $100 \%$, and the cell survival rate relative to that of the control cells is presented. The data are presented as the mean \pm SD of 3 independent experiments. (B) Cells were incubated with $2 \mu \mathrm{M}$ pemetrexed for $24 \mathrm{~h}$, and apoptosis was evaluated by green fluorescent protein-Annexin $\mathrm{V}$ and propidium iodide staining. The percentages of Annexin $\mathrm{V}$ and propidium iodide-positive cells were quantified. ${ }^{*} \mathrm{p}<0.05$ compared to the control cells. (C) Cells were treated with $2 \mu \mathrm{M}$ pemetrexed for $24 \mathrm{~h}$, after which the cell lysate was subjected to $12 \%$ SDS-PAGE to measure the expression of PARP and caspase-3, -8 and -9 . PTX, pemetrexed.

A549 cells were detectable $24 \mathrm{~h}$ after treatment with pemetrexed (Fig. 2A). Next, markers of mitochondrial dysfunction 
A

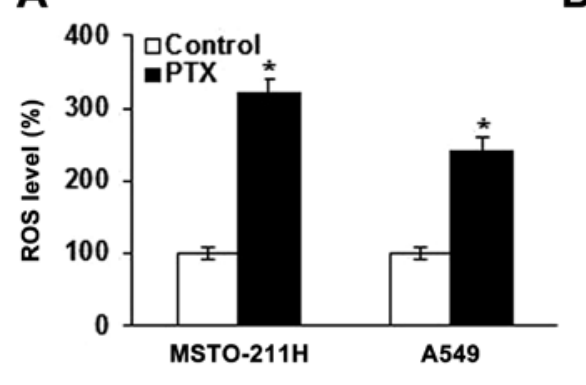

C

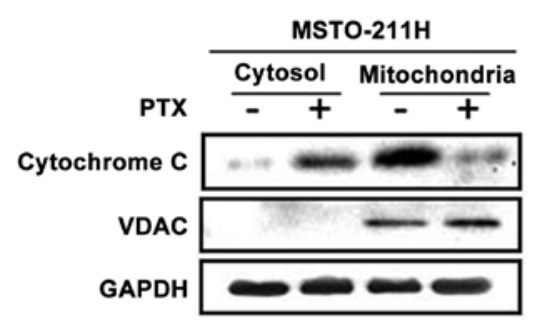

D

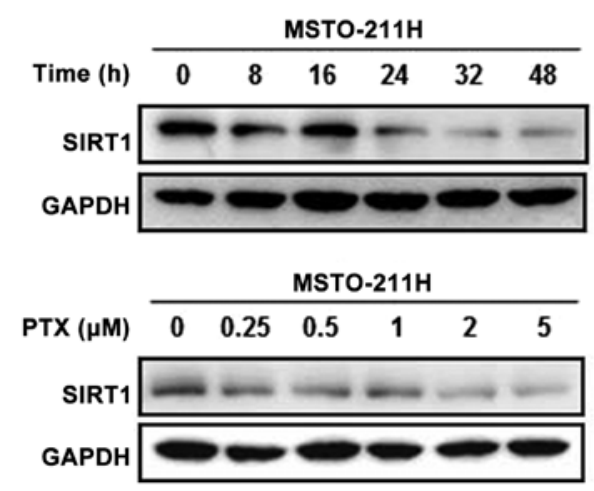

B

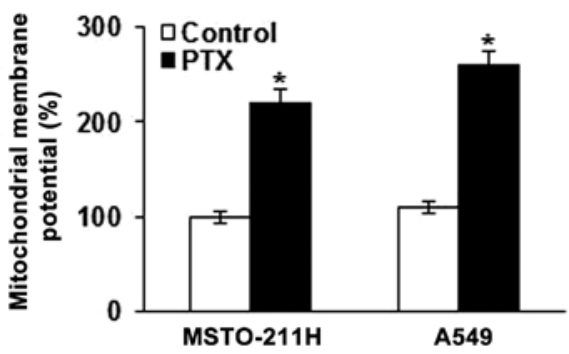

A549
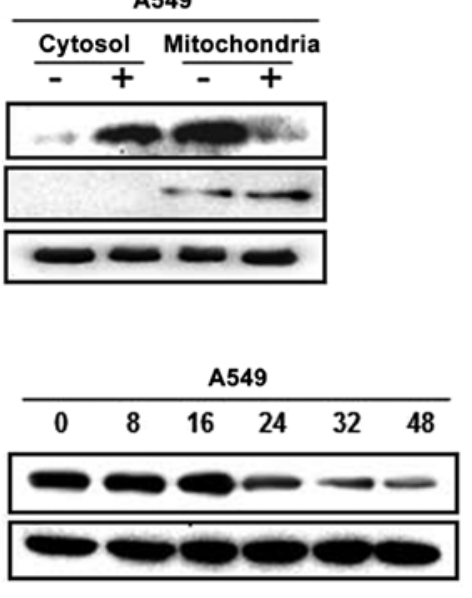

A549

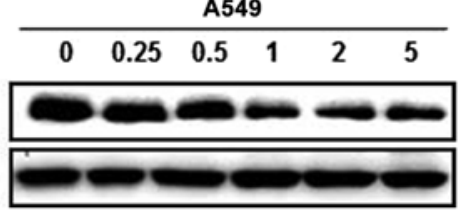

Figure 2. Contribution of ROS generation to pemetrexed-induced apoptosis and the effect of pemetrexed on SIRT1 expression. (A) Cells were treated with $2 \mu \mathrm{M}$ pemetrexed for $24 \mathrm{~h}$ and then loaded with $\mathrm{H}_{2}$ DCFDA. Fluorescence measurements were carried out with a FACSCalibur flow cytometer. The data are presented as the mean \pm SD of 3 independent experiments. ${ }^{*} \mathrm{p}<0.05$ compared to the control cells. (B) Cells were treated with $2 \mu \mathrm{M}$ pemetrexed for $24 \mathrm{~h}$, stained with $10 \mu \mathrm{g} / \mathrm{ml}$ of JC-1 and analyzed by flow cytometry. The percentage of JC-1 monomers in the control cells was set as $100 \%$, and mitochondrial membrane potential transition (MPT) relative to that of the control cells is presented. Each panel is representative of 3 identical experiments. ${ }^{\text {p }}<0.05$ compared to the control cells. (C) The cell lysate was fractionated into cytosolic and mitochondrial portions, and proteins were separated on $15 \%$ SDS-PAGE for cytochrome $c$ immunoblotting. The purity of the mitochondrial fraction was verified by western blotting with an anti-VDAC antibody. (D) The level of expression of SIRT1 was measured by western blotting. Equal protein loading was confirmed using GAPDH. Immunoblots are representative of at least 2 independent experiments. ROS, reactive oxygen species; SIRT1, sirtuin 1; VDAC, voltage-dependent anion channel. PTX, pemetrexed.

were evaluated in the cells treated with pemetrexed, including mitochondrial membrane potential transition (MPT) and cytosolic release of cytochrome $c$. JC-1 has been used to detect apoptosis induced by mitochondrial depolarization. As shown in Fig. 2B, JC-1 monomer levels were increased in the MSTO-211 and A549 cells treated with pemetrexed. Since the loss of $\Delta \Psi \mathrm{m}$ resulted in cytochrome $c$ release into the cytosol, cytochrome $c$ levels were evaluated by western blotting in the mitochondrial and cytosolic fractions (Fig. 2C). Pemetrexed was associated with increased cytosolic cytochrome $c$ and decreased mitochondrial cytochrome $c$. We next examined the effects of pemetrexed on the expression of SIRT1 in the MSTO-211 and A549 cells. SIRT1 expression was decreased in a time- and dose-dependent manner after pemetrexed treatment as compared with the control cells (Fig. 2D).

Pretreatment with NAC prevents apoptosis induced by pemetrexed. We next tested the effect of the free radical scavenger
NAC in pemetrexed-treated MSTO-211 and A549 cells. Cells were pretreated with $10 \mathrm{mM} \mathrm{NAC}$, followed by the addition of pemetrexed at $2 \mu \mathrm{M}$ for $24 \mathrm{~h}$. As shown in Fig. 3A, the enhancement of ROS generation by pemetrexed was abolished by NAC. To determine whether elevated ROS mediated the apoptosis induced by pemetrexed, the proportion of apoptotic cells was determined by Annexin V-PI staining (Fig. 3B). The proportions of Annexin V-positive MSTO-211 and A549 cells were increased by treatment with pemetrexed, whereas pretreatment with NAC markedly reduced this effect. Moreover, western blot analysis of MSTO-211 and A549 cell lysates (Fig. 3C) showed that pemetrexed decreased SIRT1 expression, enhanced the cleavage of PARP, and enhanced the expression of caspase $-3,-8$ and -9 , and pretreatment with NAC blocked these effects.

We found that JC-1 monomers were increased in the MSTO-211 and A549 cells treated with pemetrexed, whereas the loss of $\Delta \Psi \mathrm{m}$ was significantly reduced in the cells 
A

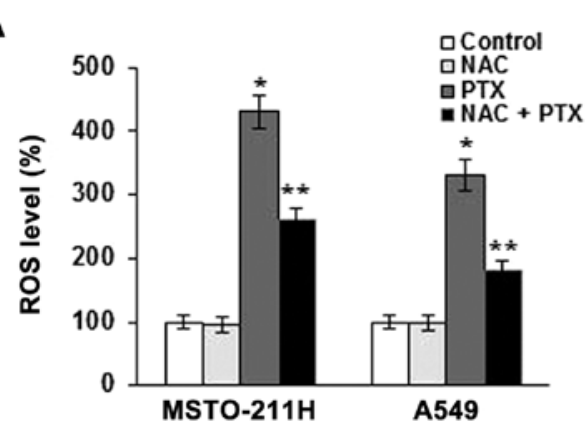

C

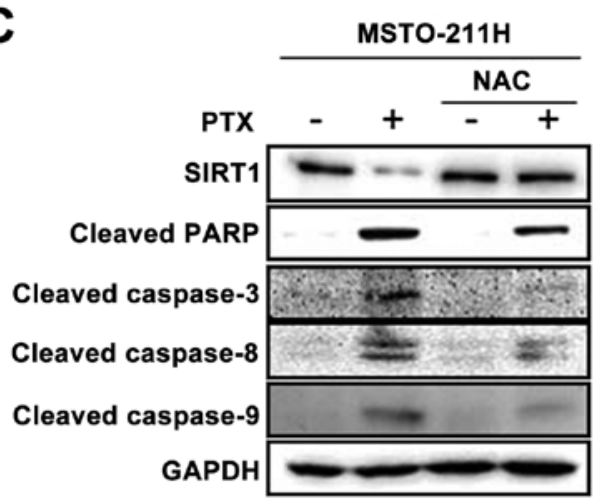

D

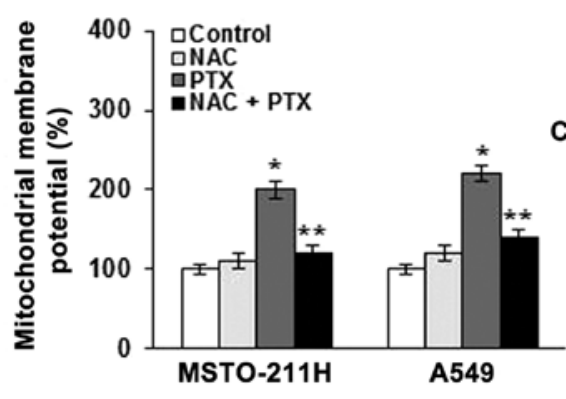

B
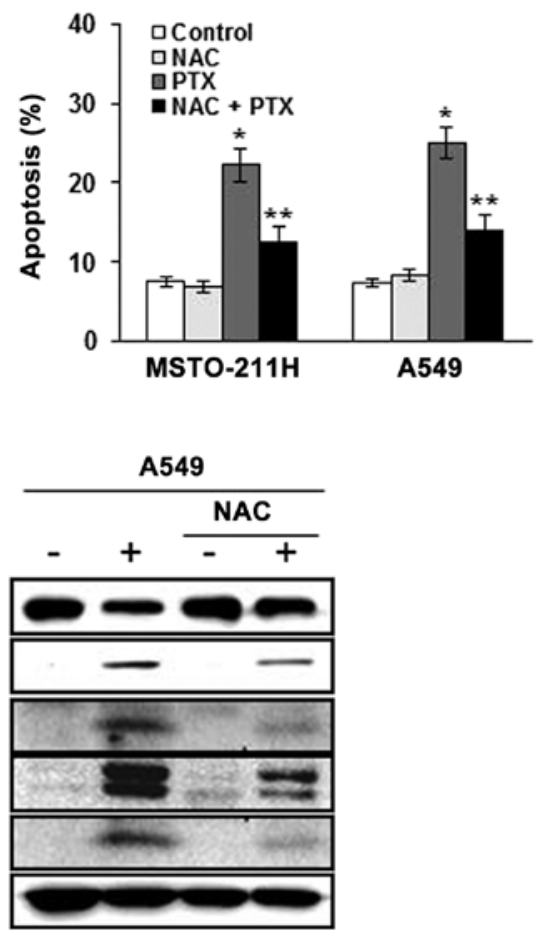

E

MSTO-211H

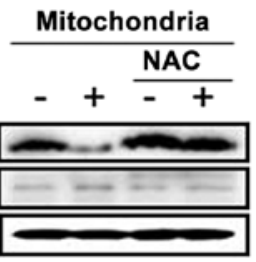

A549

Mitochondria

PTX
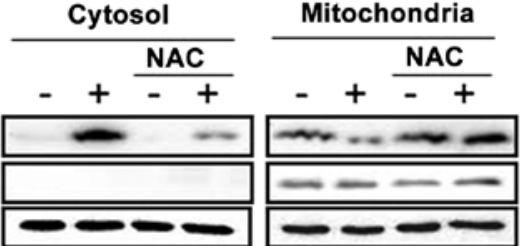

Figure 3. Effect of NAC on pemetrexed-induced apoptosis. (A) ROS generation. Cells were treated with pemetrexed in the presence or absence of $10 \mathrm{mM}$ NAC for $24 \mathrm{~h}$, and then loaded with $10 \mu \mathrm{M}$ carboxy- $\mathrm{H}_{2}$ DCFDA. Fluorescence measurements were carried out with a FACSCalibur flow cytometer. The data are presented as the mean \pm SD of 3 independent experiments ( $\mathrm{p}<0.05$ vs. untreated control; ${ }^{* *} \mathrm{p}<0.05$ vs. pemetrexed-treated cells). (B) Apoptosis was evaluated by green fluorescent protein-Annexin $\mathrm{V}$ and propidium iodide staining. The percentages of Annexin $\mathrm{V}$ and propidium iodide-positive cells were quantified ( $\mathrm{p}<0.05$ vs. untreated control; ${ }^{* *} \mathrm{p}<0.05$ vs. pemetrexed-treated cells). (C) The cell lysate was subjected to $12 \%$ SDS-PAGE to measure the expression of the indicated antibodies. Data are representative of 2 independent experiments. (D) Cells were treated with pemetrexed in the presence or absence of $10 \mathrm{mM}$ NAC for $24 \mathrm{~h}$. Cells were stained with $10 \mu \mathrm{g} / \mathrm{ml}$ of JC-1 and analyzed by flow cytometry. The percentage of JC-1 monomers of the control cells was set as $100 \%$, and MPT relative to that of the control is presented. Each panel is representative of 3 identical experiments $\left(" \mathrm{p}<0.05 \mathrm{vs}\right.$. untreated control; ${ }^{* *} \mathrm{p}<0.05 \mathrm{vs}$. pemetrexed-treated cells). (E) The cell lysates were fractionated into cytosolic and mitochondrial portions, and proteins were separated on $15 \%$ SDS-PAGE for cytochrome $c$ immunoblotting. The purity of the mitochondrial fraction was verified by western blotting with an anti-VDAC antibody. NAC, N-acetylcysteine; ROS, reactive oxygen species; MPT, membrane potential transition; VDAC, voltage-dependent anion channel. PTX, pemetrexed.

pretreated with NAC (Fig. 3D). To provide further evidence of mitochondrial dysfunction, cytosolic cytochrome $c$ was measured by western blotting in the mitochondrial and cytosolic fractions (Fig. 3E). Cytosolic cytochrome $c$ was increased by pemetrexed, and pretreatment with NAC reduced this effect. Together, these findings indicate that ROS generation plays a primary role in apoptosis induction by pemetrexed through mitochondrial dysfunction and this effect involves, at least in part, the expression of SIRT1.
Downregulation of SIRT1 augments apoptosis induced by pemetrexed. To determine the role of SIRT1 in pemetrexedinduced apoptosis in MSTO-211 and A549 cells, we knocked down SIRT1 with siRNA. The introduction of SIRT1 siRNA attenuated SIRT1 protein expression $48 \mathrm{~h}$ after transfection. No reduction in SIRT1 protein was observed in cells transfected with the scrambled siRNA, which contained the same number of each nucleotide found in the SIRT1 siRNA. As shown in Fig. 4A, the number of Annexin V-positive cells was increased 
A

MSTO-211H
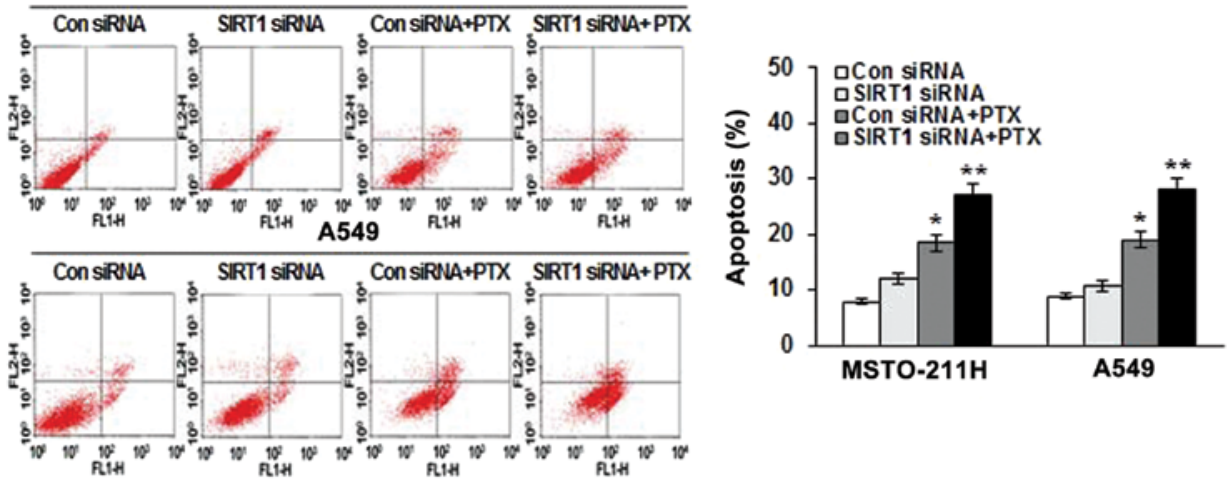

B

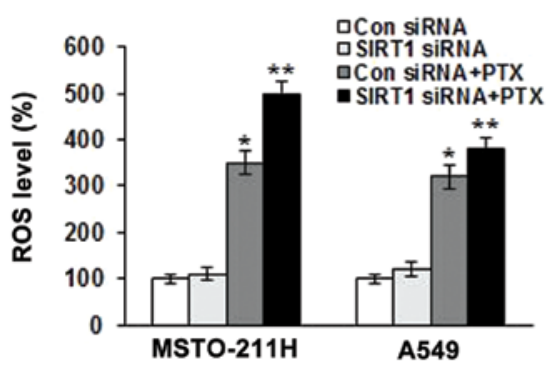

C

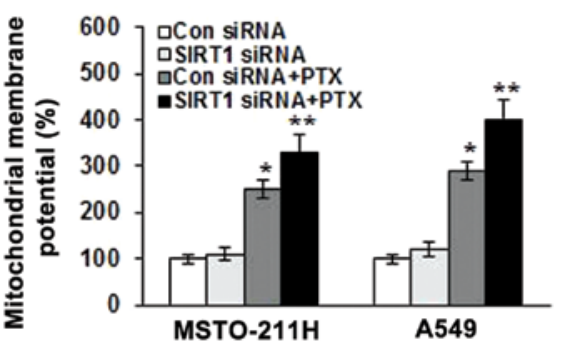

D

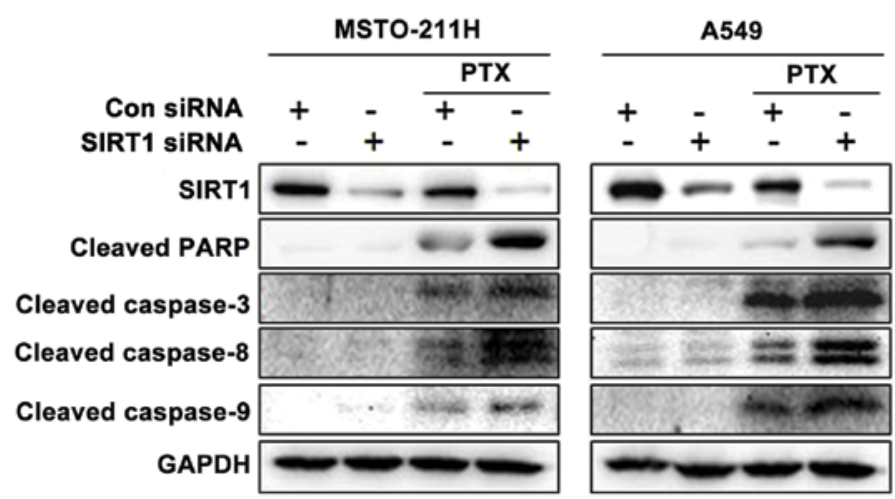

Figure 4. Effect of siRNA-mediated downregulation of SIRT1 on apoptosis induced by pemetrexed. (A) MSTO-211 and A549 cells were transfected with siRNA specific for SIRT1. Scrambled RNA containing an equivalent number of each nucleotide as the SIRT1 siRNA was used as the transfection control. Transfected cells were incubated in complete medium with or without $2 \mu \mathrm{M}$ pemetrexed for $24 \mathrm{~h}$. Apoptosis was evaluated by green fluorescent protein-Annexin $\mathrm{V}$ and propidium iodide staining. Bars represent the mean $\pm \mathrm{SD}$ of 3 independent experiments ( $\mathrm{p}<<0.05 \mathrm{vs}$. control siRNA; ${ }^{* *} \mathrm{p}<0.05 \mathrm{vs}$. control siRNA and pemetrexed-treated cells). (B) ROS generation. Cells were treated with pemetrexed in the presence or absence of SIRT1 siRNA, and then loaded with $10 \mu \mathrm{M}$ carboxy- $\mathrm{H}_{2}$ DCFDA. Fluorescence measurements were carried out with a FACSCalibur flow cytometer. The data are presented as the mean \pm SD of 3 independent experiments ( $\mathrm{p}<0.05$ vs. control siRNA; ${ }^{* *} \mathrm{p}<0.05$ vs. control siRNA and pemetrexed-treated cells). (C) Cells were treated with pemetrexed in the presence or absence of SIRT1 siRNA. Cells were stained with $10 \mu \mathrm{g} / \mathrm{ml}$ of JC-1 and analyzed by flow cytometry. The data are presented as the mean \pm SD of 3 independent experiments ( $\mathrm{p} p<0.05$ vs. control siRNA; ${ }^{* *} \mathrm{p}<0.05$ vs. control siRNA and pemetrexed-treated cells). (D) The expression levels of SIRT1, cleaved PARP, caspase- $3,-8$ and -9 were measured in the cell lysates. SIRT1, sirtuin 1. PTX, pemetrexed; ROS, reactive oxygen species.

in the SIRT1 siRNA transfectants, and combined treatment with SIRT1 siRNA and pemetrexed resulted in a greater apoptosis rate than combined treatment with the control siRNA and pemetrexed.

We next examined whether SIRT1 siRNA affects ROS generation. ROS generation in the MSTO-211 and A549 cells treated with SIRT1 siRNA and pemetrexed was increased markedly compared to that of cells treated with SIRT1 siRNA or pemetrexed alone (Fig. 4B). JC-1 monomer levels were consistently enhanced in the MSTO-211 and A549 cells treated with SIRT1 siRNA and pemetrexed (Fig. 4C). Moreover, the combination of SIRT1 siRNA and pemetrexed enhanced the expression of the processed $85-\mathrm{kDa}$ isoform of PARP, caspase-3, -8 and -9 (Fig. 4D). Together, these data indicate that downregulation of SIRT1 enhanced apoptosis induced by pemetrexed through mitochondrial dysfunction that partially involves the generation of ROS.

Resveratrol, an activator of SIRT1, attenuates the apoptosis induced by pemetrexed. We next examined whether resveratrol, an activator of SIRT1, could confer protection against the apoptosis induced by pemetrexed. As shown in Fig. 5A, pemetrexed resulted in 22.5 and $26.8 \%$, respectively, more apoptotic MSTO-211 and A549 cells than were observed in the untreated cells. However, resveratrol decreased the number of apoptotic cells after pemetrexed treatment by only 16.0 and $18.1 \%$, 

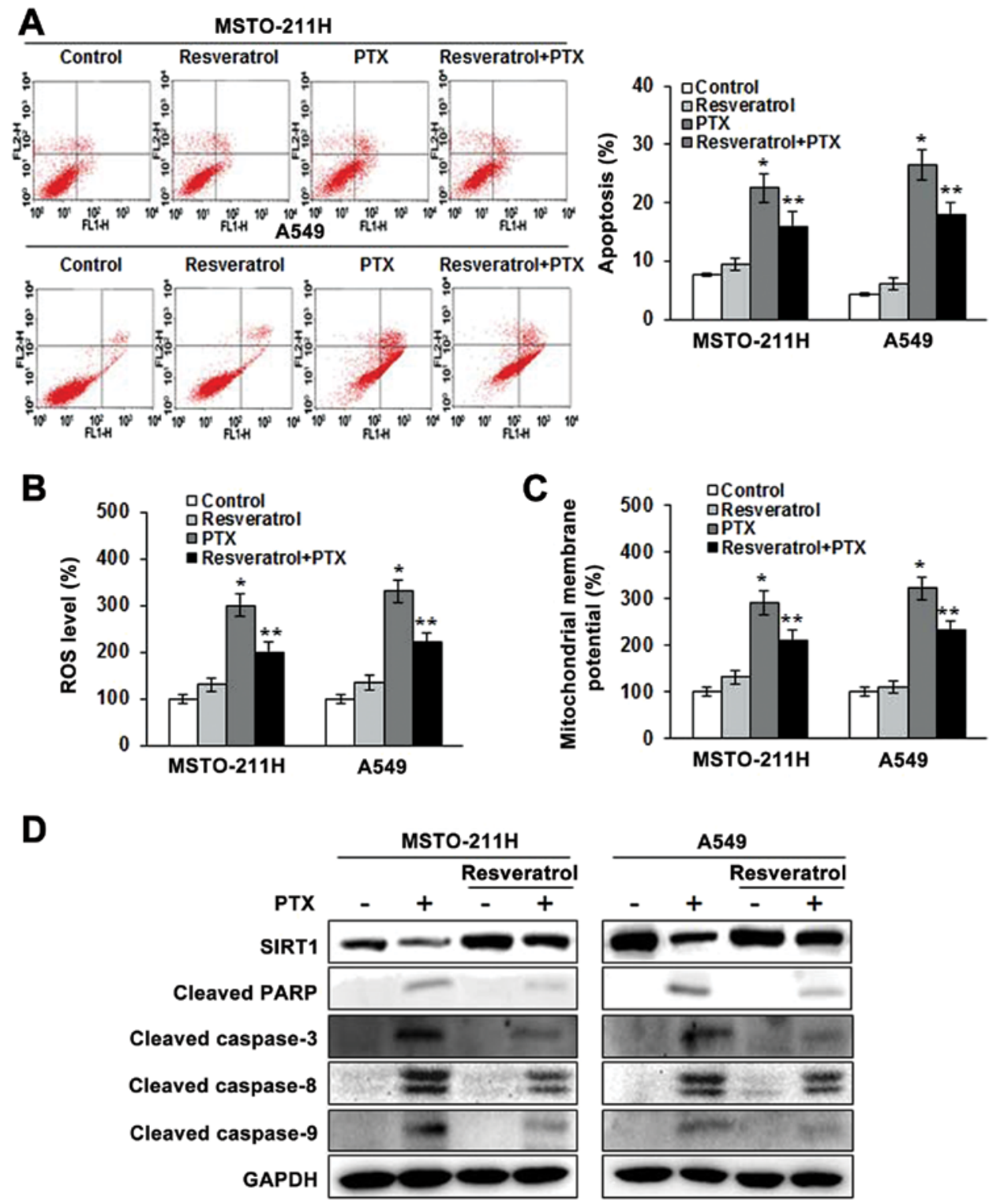

Figure 5. Effect of SIRT1 activator resveratrol on apoptosis induced by pemetrexed. (A) MSTO-211 and A549 cells were pretreated with or without $10 \mu \mathrm{M}$ resveratrol, and then further incubated in the presence or absence of $2 \mu \mathrm{M}$ pemetrexed for $24 \mathrm{~h}$. Apoptosis was evaluated by green fluorescent protein-Annexin $\mathrm{V}$ and propidium iodide staining. Bars in the histogram represent the mean $\pm \mathrm{SD}$ of 3 independent experiments $\left({ }^{*} \mathrm{p}<0.05 \mathrm{vs}\right.$. untreated control; ${ }^{* *} \mathrm{p}<0.05 \mathrm{vs}$. pemetrexed-treated cells). (B) ROS generation. Cells were treated with pemetrexed in the presence or absence of resveratrol, and then loaded with $10 \mu \mathrm{M}$ carboxy $-\mathrm{H}_{2}$ DCFDA. The data are the mean \pm SD of 3 independent experiments ( $\left(\mathrm{p}<0.05\right.$ vs. untreated control; ${ }^{* *} \mathrm{p}<0.05$ vs. pemetrexed-treated cells). (C) Cells were treated with pemetrexed in the presence or absence of resveratrol. Cells were stained with $10 \mu \mathrm{g} / \mathrm{ml}$ of JC-1 and analyzed by flow cytometry. The data are the mean \pm SD of 3 independent experiments ( ${ }^{p}<<0.05$ vs. untreated control; " ${ }^{* *}<0.05$ vs. pemetrexed-treated cells). (D) The expression levels of SIRT1, cleaved PARP, caspase- $3,-8$ and -9 were measured in the cell lysates. SIRT1, sirtuin 1; ROS, reactive oxygen species. PTX, pemetrexed.

respectively, in the MSTO-211 and A549 cells. We next examined whether SIRT1 upregulation had a role in ROS generation by pemetrexed. The results demonstrated that ROS generation in the MSTO-211 and A549 cells treated with resveratrol and pemetrexed was decreased compared to the ROS generation in cells treated with pemetrexed alone (Fig. 5B). Consistently, JC-1 monomer levels were decreased in the MSTO-211 and A549 cells treated with resveratrol and pemetrexed (Fig. 5C) as compared to those treated with pemetrexed alone. Western blot analyses showed that SIRT1 overexpression decreased the cleavage of PARP and the expression of caspase-3, -8 and -9 , even in the presence of pemetrexed (Fig. 5D). These results suggest that the reduction of apoptosis by pemetrexed is due, at least in part, to SIRT1 upregulation.

\section{Discussion}

In the present study, we examined the ability of pemetrexed to induce apoptosis in MSTO-211 malignant mesothelioma and A549 lung cancer cells, and explored the correlation between ROS generation and SIRT1 expression. We demonstrated that pemetrexed induced apoptosis by mitochondrial dysfunction in malignant mesothelioma cells and A549 NSCLC cells. These effects were mainly dependent on ROS accumulation and SIRT1 downregulation.

Previous studies have examined the effects of pemetrexed on various human tumor cells, including malignant mesothelioma and NSCLC cells. Pemetrexed as a maintenance therapy after cisplatin-based doublet chemotherapy in NSCLC 


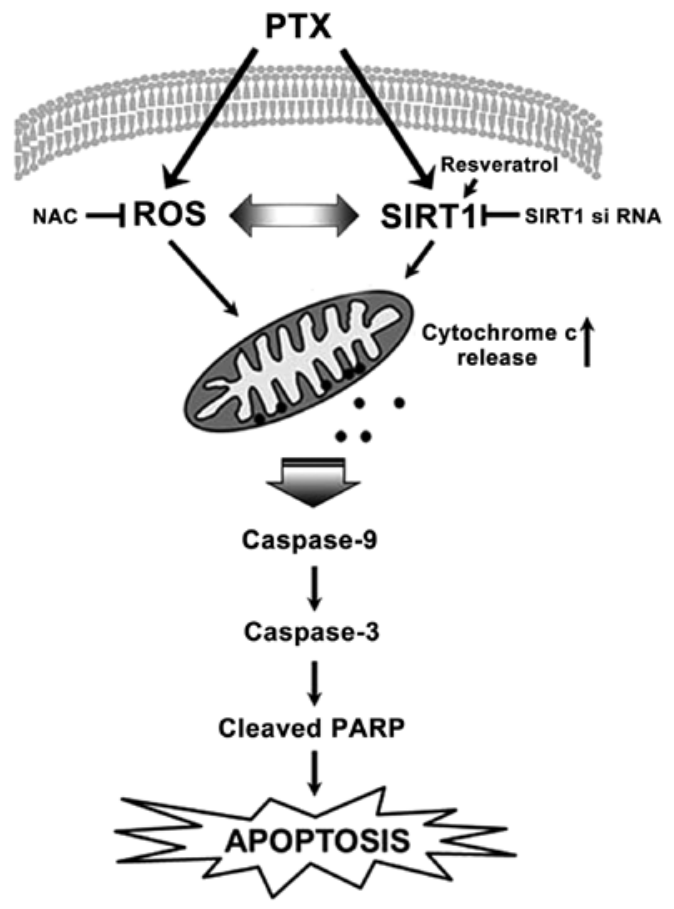

Figure 6. Schematic representation of the molecular events of pemetrexedinduced apoptosis in MSTO-211 and A549 cells. ROS production and SIRT1 downregulation induced by pemetrexed caused mitochondrial dysfunction, including the loss of mitochondrial transmembrane potential $(\Delta \Psi \mathrm{m})$ and cytosolic release of cytochrome $c$, which activates caspase-9, and subsequently leads to the activation of caspase- 3 and cleavage of PARP. ROS, reactive oxygen species; SIRT1, sirtuin 1. PTX, pemetrexed; ROS, reactive oxygen species; NAC, $\mathrm{N}$-acetylcysteine.

increases survival (17). A better understanding of the mechanisms underlying the antitumor effects of pemetrexed is needed to optimize therapeutic targeting of the drug. Pemetrexed has been shown to inhibit cell proliferation and induce apoptosis in cancer cells (18). Several studies have demonstrated that pemetrexed-induced apoptosis is closely related to caspase-dependent and -independent cascades, S-phase accumulation, deregulated activation of Akt signaling, and ataxia telangiectasia mutated/p53-dependent and -independent pathways (19-21). To date, however, the targets and anticancer mechanisms of this compound remain largely unclear.

Mitochondria play an essential role in cellular metabolism, ROS production, and regulation of cell proliferation and death $(22,23)$. A key feature of apoptosis via the mitochondrial pathway is loss of $\Delta \Psi \mathrm{m}$, followed by the release of cytochrome $c$. Cytochrome $c$ is essential to the formation of a caspase activation platform that is formed when it activates APAF-1 oligomerization, and the latter then binds to and activates caspase-9 (24-26). Our results demonstrate that the release of cytochrome $c$ into the cytosol activates caspase-9, and subsequently leads to the activation of caspase-3. Indeed, cleavage of PARP, a downstream target in this pathway, occurs during pemetrexed-induced apoptosis of malignant mesothelioma and lung cancer cells.

ROS generated in the mitochondria slow growth and cause cell cycle arrest and apoptosis (27). Many studies suggest that ROS may act as important regulators of apoptosis. ROS have been suggested to play a unique role in apoptosis regulation since they can readily produce mitochondrial dysfunction without diffusing a long distance within the cytosol (28-30). A challenge for novel treatment strategies is the fine-tuning of intracellular ROS signaling for effective therapeutic gain. Buque et al (8) reported that elevations in intracellular ROS and p53 are required for pemetrexed-induced cytotoxicity in melanoma cells. Accordingly, we investigated the possibility that ROS play a role in pemetrexed-induced apoptosis in malignant mesothelioma and lung cancer cells. We demonstrated that pemetrexed increased ROS levels. Moreover, pemetrexed-induced apoptosis, mitochondrial dysfunction and caspase activation were greatly reduced by pretreatment with NAC. These results suggest that, in this model system, ROS generation plays a primary role in the induction of apoptosis by pemetrexed.

SIRT1 promotes cytoprotection and apoptosis, depending on the nature and severity of cellular stress. SIRT1 suppresses apoptosis via modulation of a number of cell survival regulators, including p53, FOXO, HSF1, NF- $\kappa \mathrm{B}$ and DNA damage response proteins (31-33), whereas SIRT1 induces apoptosis through a number of different routes, including Nrf2, NF- $\kappa \mathrm{B}$ and p53-mediated pathways (34-36). We demonstrated that downregulation of SIRT1 by the introduction of SIRT1 siRNA into malignant mesothelioma and NSCLC cells enhanced apoptosis induced by pemetrexed. Therefore, we hypothesized that the upregulation of SIRT1 may confer protection against apoptosis induced by pemetrexed. As expected, the numbers of apoptotic malignant mesothelioma and NSCLC cells induced by pemetrexed were clearly decreased by resveratrol, an activator of SIRT1. This observation indicated that SIRT1 confers resistance against pemetrexed-induced apoptosis. In the present study, we provide for the first time a molecular mechanism for the role of SIRT1 by demonstrating that pemetrexed induces apoptosis by mitochondrial dysfunction.

In the present study, we illustrated the molecular mechanisms that mediate pemetrexed-induced apoptosis (Fig. 6). The present study confirmed that pemetrexed induced apoptosis in malignant mesothelioma and lung cancer cells through mitochondrial dysfunction by the regulation of intracellular ROS generation and SIRT1. This conclusion has both mechanistic and translational implications for future treatment strategies for patients with malignant mesothelioma and NSCLC.

\section{Acknowledgements}

The present study was supported by a grant from the Korean Health Technology R\&D Project, Ministry of Health and Welfare, Republic of Korea (A120152).

\section{References}

1. Jemal A, Siegel R, Xu J and Ward E: Cancer statistics, 2010. CA Cancer J Clin 60: 277-300, 2010.

2. Hotta K, Matsuo K, Ueoka H, Kiura K, Tabata M and Tanimoto M: Meta-analysis of randomized clinical trials comparing cisplatin to carboplatin in patients with advanced non-small-cell lung cancer. J Clin Oncol 22: 3852-3859, 2004.

3. Belli C, Fennell D, Giovannini M, Gaudino G and Mutti L: Malignant pleural mesothelioma: Current treatments and emerging drugs. Expert Opin Emerg Drugs 14: 423-437, 2009.

4. Vogelzang NJ, Rusthoven JJ, Symanowski J, et al: Phase III study of pemetrexed in combination with cisplatin versus cisplatin alone in patients with malignant pleural mesothelioma. J Clin Oncol 21: 2636-2644, 2003. 
5. Scagliotti GV, Parikh P, von Pawel J, et al: Phase III study comparing cisplatin plus gemcitabine with cisplatin plus pemetrexed in chemotherapy-naive patients with advanced-stage non-small-cell lung cancer. J Clin Oncol 26: 3543-3551, 2008.

6. Hong J, Kyung SY, Lee SP, et al: Pemetrexed versus gefitinib versus erlotinib in previously treated patients with non-small cell lung cancer. Korean J Intern Med 25: 294-300, 2010.

7. Chattopadhyay S, Moran RG and Goldman ID: Pemetrexed: Biochemical and cellular pharmacology, mechanisms, and clinical applications. Mol Cancer Ther 6: 404-417, 2007.

8. Buqué A, Muhialdin JS, Muñoz A, Calvo B, Carrera S, Aresti U, Sancho A, Rubio I and López-Vivanco G: Molecular mechanism implicated in pemetrexed-induced apoptosis in human melanoma cells. Mol Cancer 11: 25, 2012

9. Smith BC, Hallows WC and Denu JM: Mechanisms and molecular probes of sirtuins. Chem Biol 15: 1002-1013, 2008

10. Huffman DM, Grizzle WE, Bamman MM, Kim JS, Eltoum IA Elgavish A and Nagy TR: SIRT1 is significantly elevated in mouse and human prostate cancer. Cancer Res 67: 6612-6618, 2007.

11. Kim JE, Chen $\mathrm{J}$ and Lou Z: DBC1 is a negative regulator of SIRT1. Nature 451: 583-586, 2008

12. Chen WY, Wang DH, Yen RC, Luo J, Gu W and Baylin SB: Tumor suppressor HIC1 directly regulates SIRT1 to modulate p53-dependent DNA-damage responses. Cell 123: 437-448, 2005

13. Campisi J: Suppressing cancer: The importance of being senescent. Science 309: 886-887, 2005.

14. Ota H, Tokunaga E, Chang K, Hikasa M, Iijima K, Eto M, Kozaki K, Akishita M, Ouchi Y and Kaneki M: Sirtl inhibitor, Sirtinol, induces senescence-like growth arrest with attenuated Ras-MAPK signaling in human cancer cells. Oncogene 25: 176-185, 2006

15. Wolf CM and Eastman A: The temporal relationship between protein phosphatase, mitochondrial cytochrome $c$ release, and caspase activation in apoptosis. Exp Cell Res 247: 505-513, 1999.

16. Elbashir SM, Harborth J, Lendeckel W, Yalcin A, Weber K and Tuschl T: Duplexes of 21-nucleotide RNAs mediate RNA interference in cultured mammalian cells. Nature 411: 494-498, 2001.

17. Ciuleanu T, Brodowicz T, Zielinski C, et al: Maintenance pemetrexed plus best supportive care versus placebo plus best supportive care for non-small-cell lung cancer: A randomised, double-blind, phase 3 study. Lancet 374: 1432-1440, 2009.

18. Ramirez JM, Ocio EM, San Miguel JF and Pandiella A: Pemetrexed acts as an antimyeloma agent by provoking cell cycle blockade and apoptosis. Leukemia 21: 797-804, 2007.

19. Tonkinson JL, Worzalla JF, Teng CH and Mendelsohn LG: Cell cycle modulation by a multitargeted antifolate, LY231514, increases the cytotoxicity and antitumor activity of gemcitabine in HT29 colon carcinoma. Cancer Res 59: 3671-3676, 1999.

20. Chen KC, Yang TY, Wu CC, Cheng CC, Hsu SL, Hung HW Chen JW and Chang GC: Pemetrexed induces S-phase arrest and apoptosis via a deregulated activation of Akt signaling pathway. PLoS One 9: e97888, 2014
21. Yang TY, Chang GC, Chen KC, Hung HW, Hsu KH, Wu CH, Sheu GT and Hsu SL: Pemetrexed induces both intrinsic and extrinsic apoptosis through ataxia telangiectasia mutated/ p53-dependent and -independent signaling pathways. Mol Carcinog 52: 183-194, 2013.

22. Copeland WC, Wachsman JT, Johnson FM and Penta JS: Mitochondrial DNA alterations in cancer. Cancer Invest 20: 557-569, 2002

23. Kim HR, Yang SH and Jeong ET: Combination treatment with arsenic trioxide and sulindac induces apoptosis of NCI-H157 human lung carcinoma cells via ROS generation with mitochondrial dysfunction. Tuberc Respir Dis 59: 30-38, 2005.

24. Green DR and Reed JC: Mitochondria and apoptosis. Science 281: 1309-1312, 1998

25. Li LY, Luo $X$ and Wang $X$ : Endonuclease $G$ is an apoptotic DNase when released from mitochondria. Nature 412: 95-99, 2001.

26. Tournier C, Hess P, Yang DD, Xu J, Turner TK, Nimnual A, Bar-Sagi D, Jones SN, Flavell RA and Davis RJ: Requirement of JNK for stress-induced activation of the cytochrome $c$-mediated death pathway. Science 288: 870-874, 2000.

27. Burdon RH: Control of cell proliferation by reactive oxygen species. Biochem Soc Trans 24: 1028-1032, 1996.

28. Balaban RS, Nemoto S and Finkel T: Mitochondria, oxidants, and aging. Cell 120: 483-495, 2005.

29. Ozben T: Oxidative stress and apoptosis: Impact on cancer therapy. J Pharm Sci 96: 2181-2196, 2007.

30. Tan S, Sagara Y, Liu Y, Maher P and Schubert D: The regulation of reactive oxygen species production during programmed cell death. J Cell Biol 141: 1423-1432, 1998.

31. Kim EJ, Kho JH, Kang MR and Um SJ: Active regulator of SIRT1 cooperates with SIRT1 and facilitates suppression of p53 activity. Mol Cell 28: 277-290, 2007.

32. Brunet A, Sweeney LB, Sturgill JF, et al: Stress-dependent regulation of FOXO transcription factors by the SIRT1 deacetylase. Science 303: 2011-2015, 2004.

33. Westerheide SD, Anckar J, Stevens SM Jr, Sistonen L and Morimoto RI: Stress-inducible regulation of heat shock factor 1 by the deacetylase SIRT1. Science 323: 1063-1066, 2009.

34. Raynes R, Brunquell J and Westerheide SD: Stress inducibility of SIRT1 and its role in cytoprotection and cancer. Genes Cancer 4 : 172-182, 2013.

35. Kawai Y, Garduño L, Theodore $M$, Yang $J$ and Arinze IJ: Acetylation-deacetylation of the transcription factor $\mathrm{Nrf} 2$ (nuclear factor erythroid 2-related factor 2) regulates its transcriptional activity and nucleocytoplasmic localization. J Biol Chem 286: 7629-7640, 2011.

36. Yeung F, Hoberg JE, Ramsey CS, Keller MD, Jones DR, Frye RA and Mayo MW: Modulation of NF-kappaB-dependent transcription and cell survival by the SIRT1 deacetylase. EMBO J 23: 2369-2380, 2004. 